\title{
Investigation of Torsional Oscillations in Railway Vehicles
}

\author{
Tomáš Fridrichovský1,a , and Bohumil Šulc ${ }^{1}$ \\ ${ }^{1}$ Czech Technical University in Prague Faculty of Mechanical Engineering, Technická 4, 16607 Prague 6, Czech Republic
}

\begin{abstract}
The development of semiconductor electro-technics during last decades allowed us to produce railway vehicles with very high tractive power. High performances reveal new problems that were unknown until these days. One of these problems are torsional oscillations of running wheelsets, which may appear and compromise the safety of railway vehicles. This article analyzes dynamical events that may occur in drives of the vehicles and that are bound with transmission of the torque to the wheels during the operation of the vehicle, which is exposed to the variable external influences (adhesion conditions, track irregularities, variation of wheel forces, etc.). This is based on the idea of a model of simplified drive of the real railway vehicle, which is used for simulation purposes. The model serves for the parametric analysis of individual components, to make an effective design or control remedy.
\end{abstract}

\section{Introduction}

High travel velocities bring a requirement on high tractive power, which is demanded to be produced and transmitted from the traction motor to the wheels. In 2009 a slightly relative rotation between the wheel and the axle has been discovered on one of the DB (German Railways) locomotives during the maintenance. This issue has been then discovered on some other locomotives of the same type. Discovered rotation was relatively small, but it meant a big security risk due to its seriousness, especially when this problem has appeared on more vehicles. The main risk is a fact that the relative rotation between the wheel and the axle means losing friction and the failure of the whole press-fitted joint.
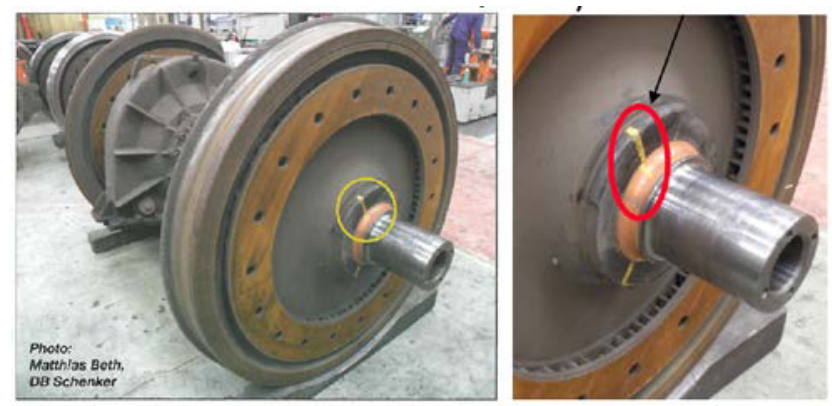

Figure 1. The detail of a relative rotation of the wheel disc on a modern electric locomotive (see yellow indication mark in the red ellipse on the right part of the picture) (source [1]).

It means that a wheel can move almost freely along the axle in transversal direction in that moment, during interaction of a guiding force. In such situation the distance between both wheels (wheelset gauge) can decrease and make vehicle derailed.

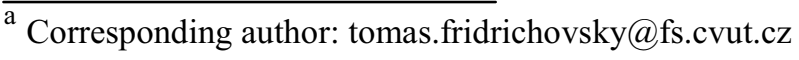

Investigation has been made when the problem has been discovered, but there weren't found any manufacture problems or failures [1]. Attention was turned to the phenomenon called "torsional oscillations of wheelsets". Torsional oscillation is a situation when both of the wheels start to oscillate one against the other (in an opposite phase). This event leads to slightly twisting of the axle.

Torsional oscillations requires an impulse to appear. This can happen when the adhesion on one of the wheels is lost. In case of a locomotive this may happen, when the vehicle runs with a high tractive effort and the adhesion force between a wheel and a rail is exceeded. Another reason is a situation when the vehicle passes through a small radius curvature. Oscillations with opposite phase may also merge during different slip velocities when the vehicle runs without tractive effort or breaking force [2]. Long lasting or periodically repeating oscillations may also lead to the creation and developing of fatigue failures in the press-fitted joint.

\section{Analysis of torsional oscillations and finding a way of their reduction}

There has been made an intensive research of sources and ways to reduction because of the seriousness of this problem. Simulation methods are a major type of the research because of experimental measurements on a real vehicle are very expensive and require a lot of time as well. Another advantage of simulation methods is a possibility to consider the wheelset as a part of the whole drive, which is represented as a controlled dynamic system. This allows to apply design processes that are based on the theory of system control. This approach has been used by Böcker, Amann and Schulz 
[3] for presentation of a design of an active reduction of torsional oscillations in a drivetrain of a car. This uses an estimation regulator with help of the Kalman filter. The research was based on a simplified and idealized interpretation of flexible and inertial properties of the used components.

Situation in railway vehicles is much more difficult. Railway vehicles work with proportionally higher values of the torque, which is influenced by much higher values of inertia momentum. External influences are specific for operation of railway vehicles (effect of wind, uncompensated centrifugal forces...), especially during creation of the model of torque transmission on the tractive effort.

One of the first publications focused on torsional oscillations was the article written by authors Kaderavek and Pernicka [1]. In this article the phenomenon was described on a general level. There was mentioned history of this phenomenon and its general specification, namely verbal description and some of the necessary conditions. More detailed description, objected on physical principle of this phenomenon, was written by authors Benker and Weber [4] who paid attention to this phenomenon in some of their articles. These articles were mainly aimed on a wheelset that was presented as a force excited mechanical oscillator.

Possibility of detection of the oscillations has been described by authors Markovic, Kostic and Bojovic [5]. Authors made a simplified model of the class 444 locomotive, propelled via DC motors. The model was created as a scheme of transmission of the torque, using the set of parts connected via torsional elastic elements, representing the wheelset, the gearbox and the traction motor. Results of the simulations showed that in the moment when the adhesion is decreased enough and the wheelset starts to slip, the voltage of the motor increases above its nominal value. Closer look at the voltage course shows that the voltage oscillates with a small amplitude and a specific frequency. This frequency corresponds to the natural frequency of the wheelset and appears only in the moment of losing the wheelset adhesion.

Possibility of reduction of the oscillations has been described by authors Bieker, Dede, Dörner, Klein and Pusnik [6]. Authors have dealt with an idea of using of brake discs elastically connected to the wheels that may serve as additional oscillators. These oscillators, suitably configured, should start to oscillate instead of the wheelset when the adhesion is lost. This means the axle shouldn't twist at all. Then brake discs can serve as absorbers of the wheelset torsional oscillations. This may work only on condition when all the parameters are well set. This may be problematic because of diameter of the wheel that may differ during the vehicles lifetime, which causes changes of the wheelset natural frequency. Another problem is a fact that the natural frequency depends on the value of tangential forces between the wheel and the rail, the concept of the drive, and velocity of the vehicle $[2,7,8]$.

\section{Idealization used in presented approach to the modelling of torsional oscillations}

The problem seems to be related only to the wheelset, but there are more factors that may have influence on it. These factors are necessary to be considered because of complexity of the models, in simplified form with certain degree of idealization or, because of difficulty of mathematical description, fully neglected. For example a vehicle body may interact with an elastically connected two-axle bogie. The wheel force, interacting between the wheel and the rail, and the pulling force may then differ during the operation. These influences are not considered in the presented model, which is used for further analysis. The major attention has been aimed on the model of the whole drive chain and the adhesion between the wheel and the rail.
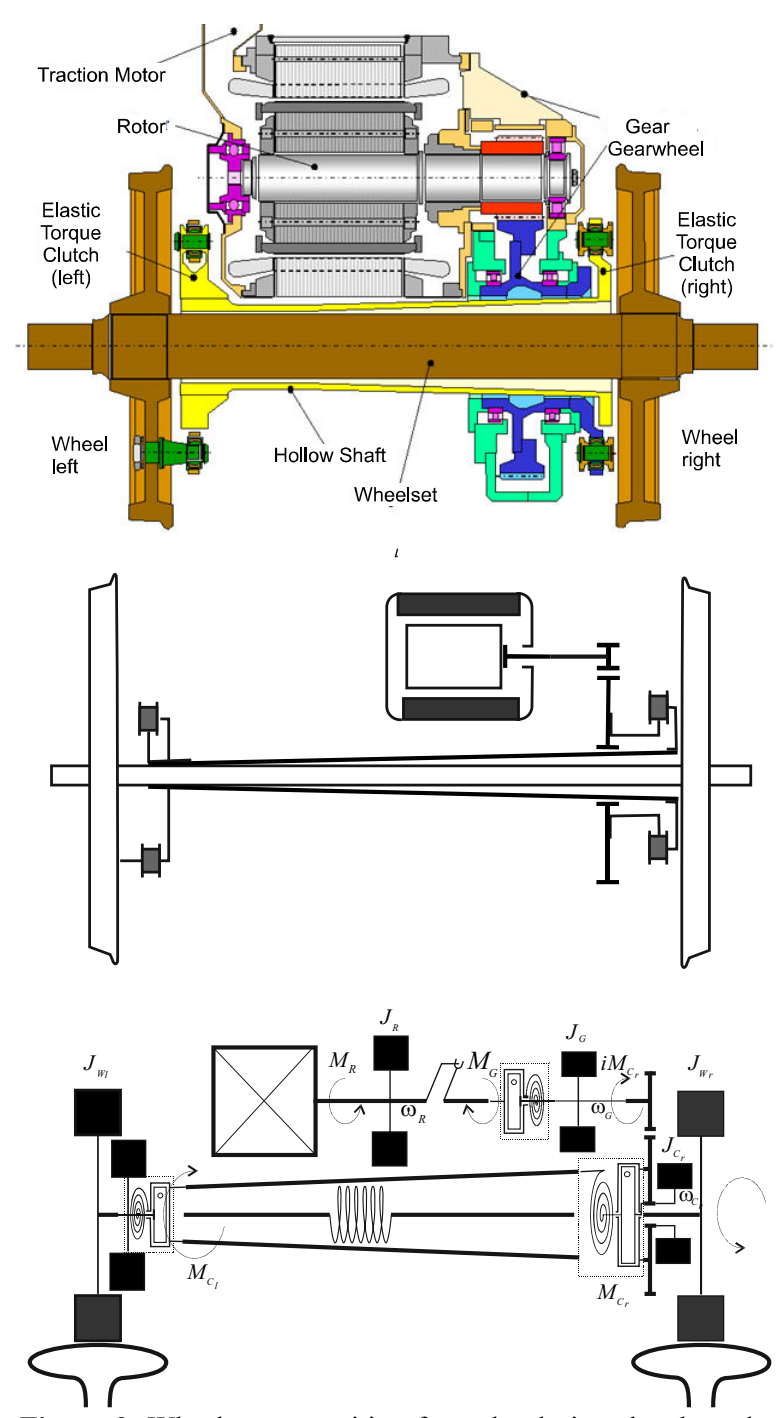

Figure 2. Wheelset - transition from the design sketch to the substitute idealized concept. 


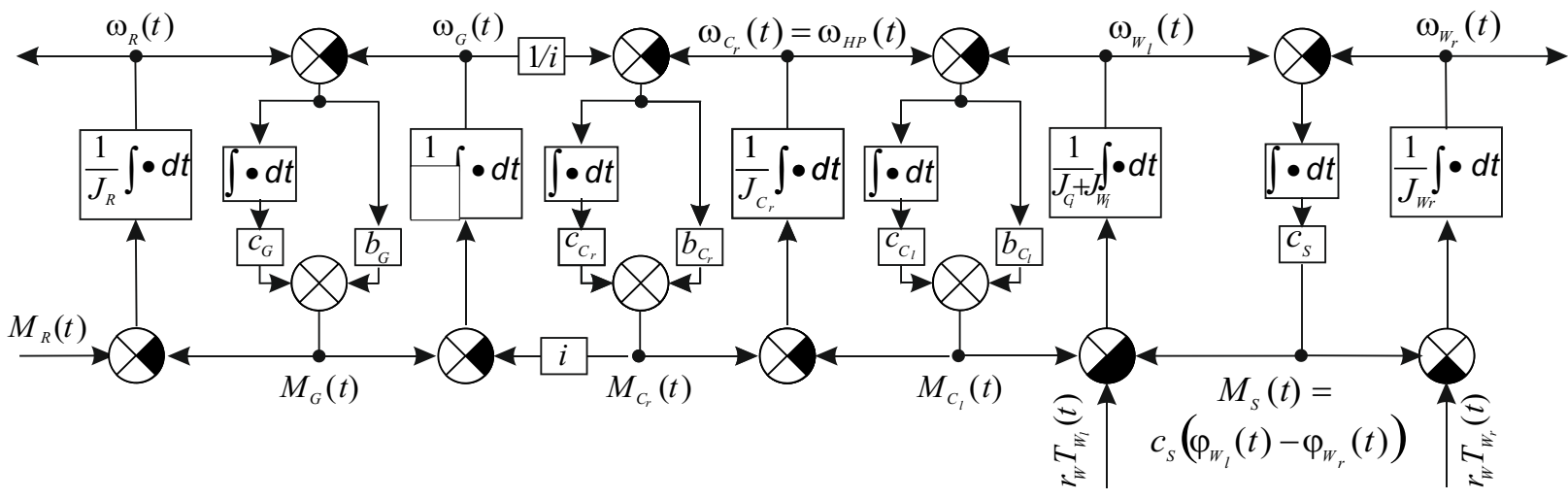

Figure 3. Block scheme of the model of the torque transmission from the traction motor to the wheels.

Design scheme of the drive chain containing cross section view is shown on Figure 2. As a support of the models creation, the decomposition on the base elements has been made as it can be seen in the mid part of Figure 2. The drive chain is here divided into fundamental parts, namely a traction motor, a gearbox, clutches, a hollow shaft, an axle and wheels.

Schematically shown substitute concept of the torque transmission, in the lower part of the image is the fundamental part of the creation of the mathematical model. The components, which are passing a rotary motion, are idealized and their mechanical properties are centralized into fundamental elements. These elements represent moments of inertia, torsional stiffness and torsional damping. The two rails on the bottom of the picture represent a requirement to include the adhesion model.

The model of drive chains dynamics is composed of three connected subunits:

- model of transmission of the torque on the wheelset including the model of the gearbox

- model of transmission of the toque on the adhesion force between the wheel and the rail

- model of the traction motor

The creation of the model was based on the idea that the schematically shown mechanical components are massless and rigid and are unable to resist to the rotary motion. All of these resists are made via connected elements that represent these concentrated properties.

These elements are resolved with different line thickness or bordered with the dashed line on the Figure 2. Continuously distributed properties (moment of inertia, torsional stiffness, torsional damping) are replaced with three idealized elements: massless spiral spring with a constant torsional stiffness c, rotary hydraulic dumper with an incompressible fluid with the dumping coefficient $b$, rotating disc with the moment of inertia J. These three elements create a fictional component that is connected into the modelled component of the drive, so it is not disrupting the functionality of the transmission of the rotary motion. The respective component is then considered as ideally rigid and massless.
The equation description, which is based on the mentioned substitute concept, is a shortened description of the increment interpretation of all variables in the symbolic labelling of all the values that are characterizing the rotary motion (the angle of rotation $\varphi(t)$, the rate of its change $\omega(t)=\dot{\varphi}(t))$. It means all the values are the increments - deviations from the nominal values. The usually used symbol $\Delta$ has been suppressed because of shortening of the description.

The increment model of the drive is described with following equations. Symbols in brackets show where is the value belonging to - rotor $(\mathrm{R})$, gearbox $(\mathrm{G})$ right torsional clutch $\left(\mathrm{C}_{\mathrm{r}}\right)$ hollow shaft $(\mathrm{HP})$, left wheel $\left(\mathrm{W}_{1}\right)$, the axle $(\mathrm{S})$ and right wheel $\left(\mathrm{W}_{\mathrm{r}}\right)$.

$$
\begin{gathered}
J_{R} \dot{\omega}_{R}(t)=M_{R}(t)-M_{G}(t) \\
M_{G}(t)=c_{G}\left(\varphi_{R}(t)-\varphi_{G}(t)\right)+b_{G}\left(\omega_{R}(t)-\omega_{G}(t)\right) \\
J_{G} \dot{\omega}_{G}(t)=M_{G}(t)-i M_{C_{r}}(t) \\
M_{C_{r}}(t)=c_{C_{r}}\left(\frac{1}{i} \varphi_{G}(t)-\varphi_{C_{r}}(t)\right)+b_{C_{r}}\left(\frac{1}{i} \omega_{G}(t)-\omega_{C_{r}}(t)\right) \\
J_{C_{r}} \dot{\omega}_{C_{r}}(t)=M_{C_{r}}(t)-M_{H P}(t) \\
\varphi_{H P_{r}}(t)=\varphi_{H P_{l}}(t)=\varphi_{H P}(t) \quad \omega_{H P_{r}}(t)=\omega_{H P_{l}}(t)=\omega_{H P}(t)
\end{gathered}
$$

$\varphi_{H P_{r}}(t)=\varphi_{H P_{l}}(t)=\varphi_{H P}(t) \quad \omega_{H P_{r}}(t)=\omega_{H P_{l}}(t)=\omega_{H P}(t)$

$M_{C_{l}}(t)=c_{C_{l}}\left(\varphi_{H P}(t)-\varphi_{W_{l}}(t)\right)+b_{C_{l}}\left(\omega_{H P}(t)-\omega_{W_{l}}(t)\right)$

$\left(J_{W_{l}}+J_{C_{l}}+\frac{1}{2} J_{S}\right) \dot{\omega}_{W_{l}}(t)=M_{C_{l}}(t)-M_{W_{l}}(t)$

$M_{S}(t)=c_{S}\left(\varphi_{W_{l}}(t)-\varphi_{W_{r}}(t)\right)$

$\left(J_{W r}+\frac{1}{2} J_{S} \dot{\omega}_{W_{r}}(t)=M_{C_{l}}(t)-r_{W} T_{W_{r}}(t)-M_{S}(t)\right.$ 
Dynamic changes of the angular velocities of the wheels make changes of the tangential forces which depend on them.

The model of the transmission of the tangential forces between the wheel and the rail is based on Polach theory [9]. Tangential forces are transmitted via friction which is bounded with a slight slip of the wheels on the rails. The amount of friction and the tangential forces as well is based on a friction coefficient $\mu$.

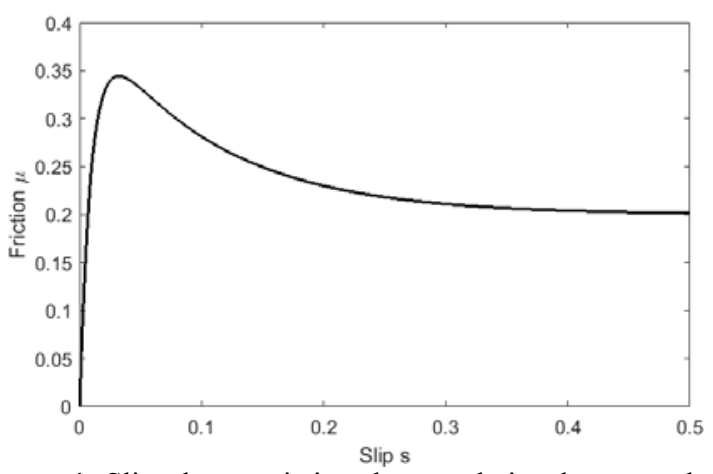

Figure 4. Slip characteristics shows relation between the slip and the friction. The characteristics is described via eq. (11).

The relation between the slip and the friction is expressed via slip characteristics, which has been analytically described for the simulation purposes with a sum of two exponential functions.

$$
\mu(s)=\mu_{M A X}\left(1-e^{\frac{-s}{\tau_{1}}}\right)-\mu_{R E D} h(s-0,01)\left(1-e^{-\frac{(s-0,01)}{\tau_{2}}}\right)
$$

The variability of the slip conditions for a dry or wet rail may be well expressed with appropriate chosen parameters $\mu_{\text {MAX }}, \mu_{\text {RED }}, \tau_{1}, \tau_{1}$. The slip $s$ is defined as a dimensionless ratio of a perimeter speed of the wheel and the vehicle velocity $v(t)$ and is described with the following equation

$$
\Delta s(t)=\frac{r_{w} \Delta \omega_{w}(t)-\Delta v(t)}{r_{w} \omega_{w 0}}
$$

This adhesion model is based on an assumption that the vehicle is in a steady state. This means the vehicle moves with a constant velocity $v_{0}$, the pulling force of the vehicle, divided on single wheels is constant as well as the tangential forces $T_{w 0}$, which correspond with the engine torque $M_{w 0}$. Then the slip must have a steady value $s_{0}$ so the friction coefficient $\mu_{0}=g^{-1} T_{w 0} / m_{w}$ corresponds with the requested value of the tangential force $T_{w 0}$ coinciding with the vehicle mass divided onto single wheels $m_{w}$.

The angular velocity of the wheel differs by an increment in the non-steady state. This velocity is bounded with an angular acceleration and a moment of inertia $J_{w}$ during imbalance of the dynamic change of the torque on the wheel $\Delta M_{w}(t)$, transmitted through the drive, and change of the torque that occurs during changes of the slip $m_{w} g \Delta s(t)$.

The calculation of the slip differences is then necessary to be completed with a dynamics of the velocity changes that depends on drive resists. The drive resist (the roll and the aero dynamical resist) can be expressed with a parabolic relation for a single vehicle or the whole train with an equation $m_{s}\left(a+b v(t)+c v^{2}(t)\right)$. This drive resist is necessary to be linearized according to an increment form of the model and then divided into single drives or wheels.

The model of the asynchronous motor is based on a real traction motor type ML $4550 \mathrm{~K} / 6$ whose parameters are following

$\begin{array}{lll}\text { Power output } & 1600 & \mathrm{~kW} \\ \text { Nominal speed } & 1825 & \mathrm{~min}-1 \\ \text { Maximum torque } & 10000 & \mathrm{Nm} \\ \text { Nominal torque } & 8400 & \mathrm{Nm}\end{array}$

The complex model of the traction motor has been replaced with a linear approximation of the relation between the torque and speed due to the fast dynamics of the initial simulations.

\section{Results}

The experiments were focused on the situation when the torque is rapidly changed from its steady state. This corresponds to the situation when the adhesion is decreased and the stick slip protection makes an intervention (the torque of the motor is decreased or increased in this moment).
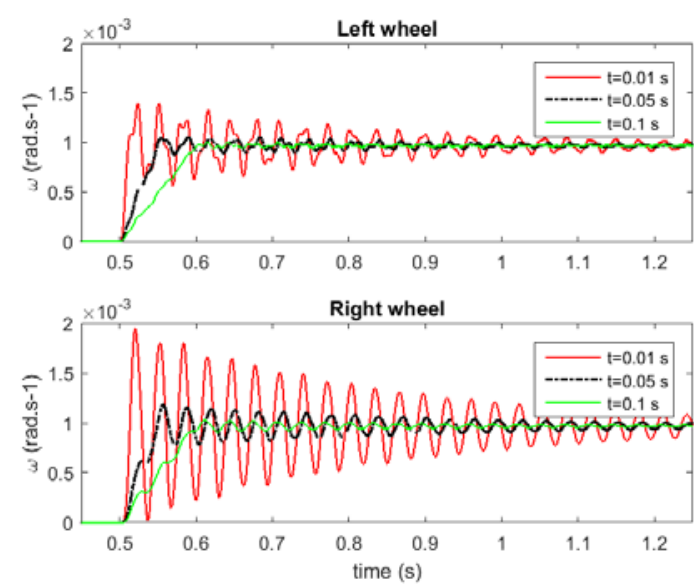

Figure 5. Time courses of the oscillations during change of the motor torque. Torque increment is $500 \mathrm{~N} . \mathrm{m}$.
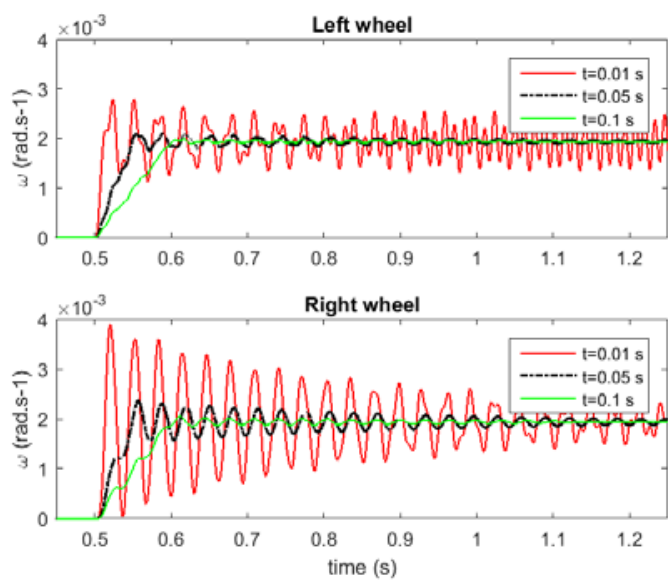

Figure 6. Time courses of the oscillations during change of the motor torque. Torque increment is $1000 \mathrm{~N} . \mathrm{m}$ 

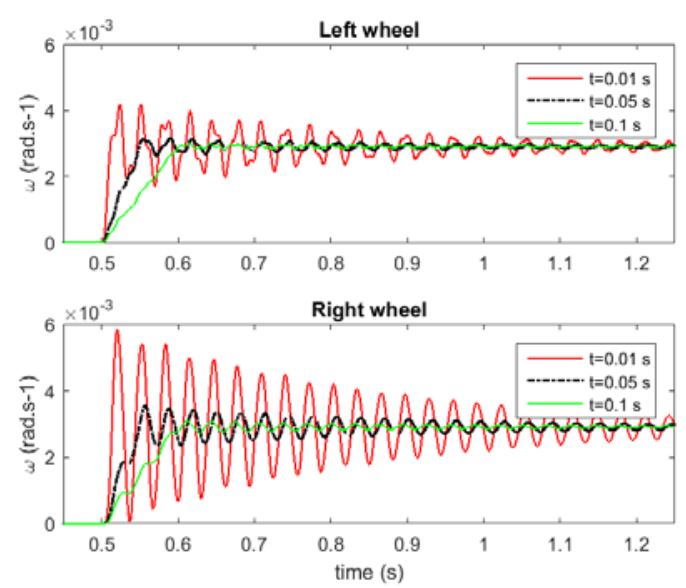

Figure 7. Time courses of the oscillations during change of the motor torque. Torque increment is $1500 \mathrm{~N} . \mathrm{m}$

The simulations took into account different size of the actual torque changes and different speed of these changes. Enclosed figures show following

- Faster the speed of the change is, the greater the amplitudes of the oscillations are.

- Higher the change of the torque is, the greater the amplitudes of the oscillations are.

These oscillations have relatively small amplitudes but they may be dangerous when the torque control changes the value of the torque repeatedly in quick during the operation of the vehicle. As a result, this may lead to very high oscillations created in the drive of the vehicle.

\section{Conclusions}

From a series of experiments that have been carried out are presented those focused on the angular velocities of the wheels. These velocities can be expected as a possible consequences of a rapid change of the motor torque. The motor torque is the most important and easiest exploitable way how to supress possible occurrence of oscillations by means of control circuit using the motor torque as a manipulated variable.

Created simulation model includes a model of running behaviour that is respecting change of the adhesion conditions and detailed model of the asynchronous motor. This allows to study the influence of the level of knowledge of the known parameters. This is then used to assess an impact of different design settings or control actions required for reduction of the torsional oscillations.

\section{Acknowledgement}

The paper was created with the financial support SGS grant No. SGS14/184/OHK2/3T/12 and of the Technology Agency of the Czech Republic, project No TE01020038 "Competence Centre of Railway Vehicles".

\section{References}

1. P. Kaderavek, J. Pernicka, Torsion oscillations of powered wheelsets, Railvolution, 34-37 (2013)

2. J. Kolář, Design of a Wheelset Drive, Transaction on Electrical Engineering, 4, 11-19 (2015)

3. J. Böcker, N. Amann, B. Schulz, Active Suppression of Torsional Oscillations, EEE/ASME Transactions on Mechatronics, 9, 6 (2004)

4. T. Benker, T. Weber, Torsionsschwingungen von Raddsätzen - eine Herausforderung?, Eisenbahningenieur, 47-52 (2015)

5. P. Markovic, D. Kostic, N. Bojovic, One Method for Detection of Torsional Oscillations of Driving Axles of Electrical Locomotives, Proceedings of the 22nd international conference on Current problems in Rail vehicles - PRORAIL 2015, 1, 27-35 (2015)

6. G. Bieker, J. Dede, D. Dörner, H. Klein, A. Pusnik, Bremsscheibe als Tilger für Radsatztorsionsschwingungen, ZEV Rail, 381-387 (2014)

7. J. Kolár, Problems with modellling of the influence of the vertical track irregularities on the dynamics of the vehicle drive, Proceedings of the 22nd international conference on Current problems in Rail vehicles - PRORAIL 2015, 1, 275-286 (2015)

8. J. Kolár, Dynamics of Wheelset with Axle Gearbox, Proceedings of the 22nd international conference on Current problems in Rail vehicles PRORAIL 2015, vol. 2, 139-148 (2011)

9. O. Polach, Creep forces in simulations of traction vehicles running on adhesion limit, Proceedings of the 6th International Conference on Contact Mechanics and Wear of Rail/Wheel Systems (CM2003), 279-285 (2003) 